\title{
Clinical utility of the KAMRA corneal inlay
}

This article was published in the following Dove Press journal:

Clinical Ophthalmology

18 May 2016

Number of times this article has been viewed

\author{
Shehzad Anjam Naroo \\ Paramdeep Singh Bilkhu \\ Ophthalmic Research Group, School \\ of Life \& Health Sciences, Aston \\ University, Birmingham, UK
}

\begin{abstract}
The treatment of presbyopia has been the focus of much scientific and clinical research over recent years, not least due to an increasingly aging population but also the desire for spectacle independence. Many lens and nonlens-based approaches have been investigated, and with advances in biomaterials and improved surgical methods, removable corneal inlays have been developed. One such development is the KAMRA ${ }^{\mathrm{TM}}$ inlay where a small entrance pupil is exploited to create a pinhole-type effect that increases the depth of focus and enables improvement in near visual acuity. Short- and long-term clinical studies have all reported significant improvement in near and intermediate vision compared to preoperative measures following monocular implantation (nondominant eye), with a large proportion of patients achieving Jaeger (J) 2 to J1 ( $~ 0.00 \log$ MAR to $\sim 0.10 \log$ MAR) at the final follow-up. Although distance acuity is reduced slightly in the treated eye, binocular visual acuity and function remain very good (mean $0.10 \log \mathrm{MAR}$ or better). The safety of the inlay is well established and easily removable, and although some patients have developed corneal changes, these are clinically insignificant and the incidence appears to reduce markedly with advancements in KAMRA design, implantation technique, and femtosecond laser technology. This review aims to summarize the currently published peer-reviewed studies on the safety and efficacy of the KAMRA inlay and discusses the surgical and clinical outcomes with respect to the patient's visual function.
\end{abstract}

Keywords: presbyopia, refractive surgery, implants, cornea

\section{Introduction}

Treatment for the correction of presbyopia has continued to be the focus of considerable research. Typically affecting people from 40 years of age, the loss of near visual acuity is often attributed to increased lens nucleus hardness and subsequent inability of the lens capsule to compress the lens to a more convex state over time. ${ }^{1-3}$ However, as lens thickness increases with age, the space between the lens and ciliary body reduces, and the angle of zonule insertion may change and therefore render ciliary body contraction ineffective. ${ }^{4-6}$ Presbyopia can significantly impact the quality of life and combined with an increasingly aging global population it poses a greater demand for spectacle independence. ${ }^{7}$

Approaches to treat presbyopia have included the use of intracorneal inlays to either change the refractive power of the cornea based on corneal multifocality ${ }^{8}$ or increase the refractive power of the central cornea by changing its curvature. ${ }^{9,10}$ Another inlay method which has been studied in great detail is the use of small-aperture optics to increase the depth of focus based on the pinhole effect. ${ }^{11,12}$ This commercially available inlay is known as the KAMRA ${ }^{\mathrm{TM}}$ inlay (AcuFocus Inc., Irvine, CA, USA), and this review aims to summarize the efficacy and safety of currently published clinical studies of this procedure.
Correspondence: Shehzad Anjam Naroo Ophthalmic Research Group, School of Life \& Health Sciences, Aston University, Birmingham B4 7ET, UK

Tel +44 I2I 2044132

Fax +44 I 2 I 2044048

Email s.a.naroo@aston.ac.uk
Clinical Ophthalmology 2016:10 9/3-9/9

(c) (1) (-) ๑ 2016 Naroo and Bilkhu. This work is published and licensed by Dove Medical Press Limited. The full terms of this license are avalabble at https://www.dovepress.com/terms.php cc. hereby accept the Terms. Non-commercial uses of the work are permitted without any further permisision from Dove Medical Press Limited, provided the work is properly atributed. For permision for commercial use of this work, please see paragraphs 4.2 and 5 of our Terms (htpps://www.dovepress. com/terms.php).
Dovepress

http://dx.doi.org/10.2147/OPTH.S89132
13

son




\section{Methodology}

Clinical trials of the KAMRA inlay used in this literature review were searched in PubMed using the following keywords alone and in combination (where appropriate): KAMRA, corneal inlay, safety, efficacy, and visual outcomes. In total, 14 clinical trials were identified and used for analysis.

\section{The KAMRA inlay}

The KAMRA design (ACI7000PDT) consists of a $3.8 \mathrm{~mm}$ diameter microperforated $(8,400$ holes $5-11 \mu \mathrm{m}$ in diameter) tinted disc with $1.6 \mathrm{~mm}$ central aperture at $6 \mu \mathrm{m}$ thick and is made of polyvinylidene fluoride and carbon nanoparticles. Figure 1 shows the size of the KAMRA inlay compared to a $14 \mathrm{~mm}$ soft contact lens. The inlay is designed to be inserted in the line if sight of the nondominant eye and implanted in a femtolaser created corneal lamellar pocket at least $220 \mu \mathrm{m}$ deep. Figure 2 shows a schematic of the inlay design.

The inlay is designed to allow light to enter through the central aperture, thus reducing retinal image blur and increasing depth of focus to allow increased near and intermediate visual acuity. As the inlay does not split light between different focal points, this allows the patient to maintain binocular summation. ${ }^{13}$ Figure 3 shows the inlay in situ in a patient's cornea.

Given that this is an additive procedure (ie, no corneal tissue is removed), it can be combined with refractive laser vision correction procedures where the eyes are made emmetropic - here the inlay is situated in a lamellar pocket at least $100 \mu \mathrm{m}$ beneath the initial laser in situ keratomileusis (LASIK) flap. ${ }^{14}$ Further, it can be implanted in previously pseudophakic eyes, which has been shown, albeit in a few cases, to produce a significant improvement in near acuity without affecting distance acuity. ${ }^{15}$ Based on an eye model, it has been suggested that the best depth of focus is achieved

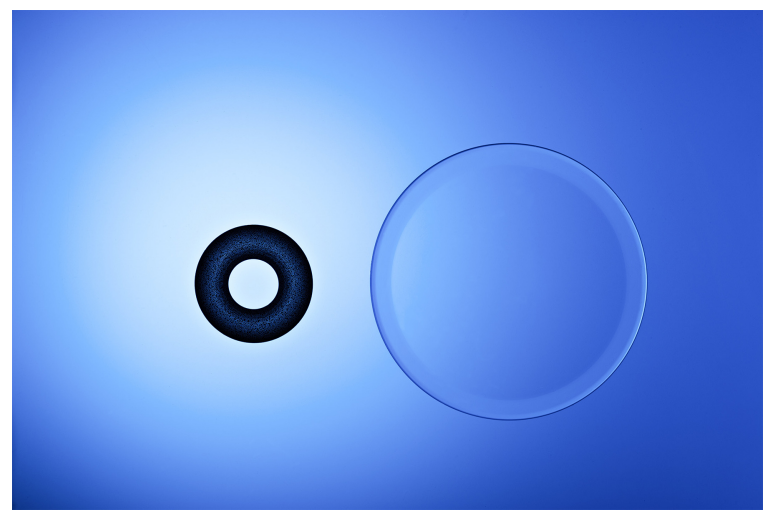

Figure I The size of the KAMRA inlay compared to a $14 \mathrm{~mm}$ diameter soft contact lens.

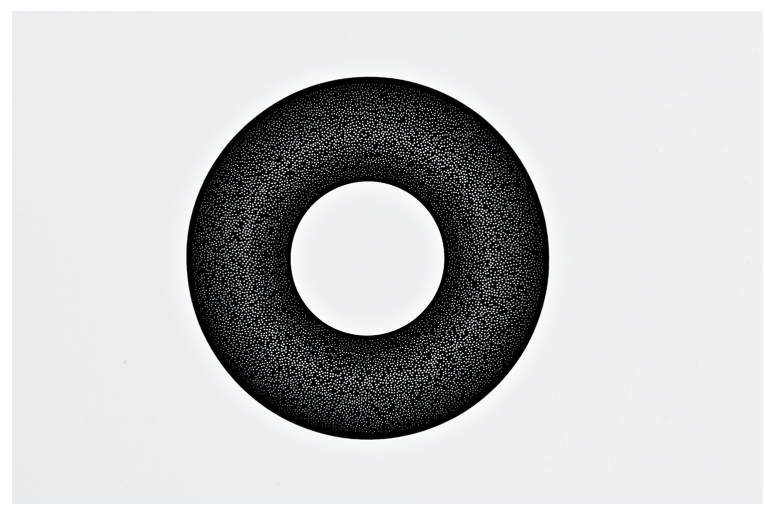

Figure 2 A schematic of the KAMRA inlay design.

where the dominant eye is made plano and the nondominant eye is made myopic $(-0.75$ to $-1.00 \mathrm{D}){ }^{16}$

\section{Clinical performance}

The efficacy of the KAMRA inlay has been investigated in several studies, albeit in case series where pre- and postoperative measures were compared rather than case-control clinical studies. Nonetheless, all have reported significant improvements in near visual acuity following implantation. However, it should be borne in mind by the reader that all currently published studies are company sponsored (AcuFocus).

In a study comprising hyperopic, myopic, and emmetropic patients (180 patients), the KAMRA inlay (model ACI7000PDT) was implanted in the nondominant eye together with a bilateral LASIK for the ametropic patients. Although only 64 patients were available for follow-up, the KAMRA-treated eye resulted in a seven-line improvement in $\operatorname{logMAR}$ uncorrected near visual acuity (UNVA) in hyperopic eyes (to mean of $0.18 \log$ MAR), two lines in myopic eyes (0.12 logMAR), and six lines in emmetropic eyes $(0.10 \log$ MAR $)$ after 6 months. ${ }^{14}$ The smaller

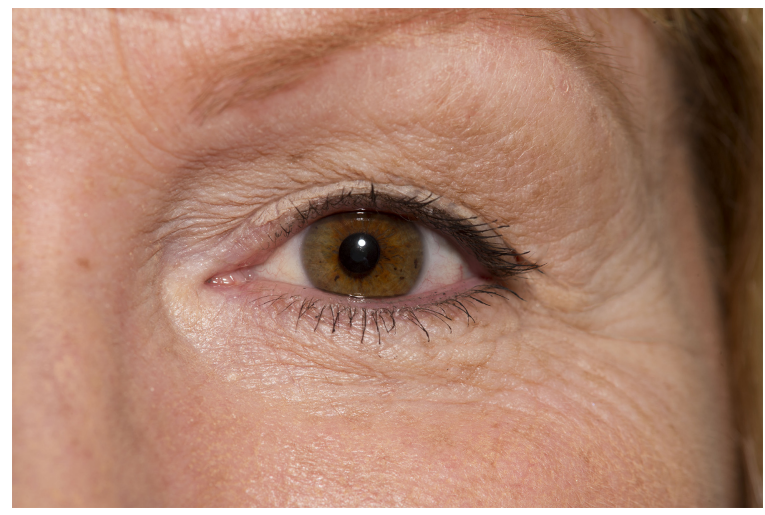

Figure 3 The KAMRA inlay inserted in a patient's cornea. 
improvement in myopic eyes was not unexpected due to preoperative good UNVA, and this was reflected in the patient satisfaction scores for this myopic group where the improvement in overall vision was not statistically significant. ${ }^{14}$ Uncorrected distance visual acuity (UDVA [logMAR]) also improved in the treated eye, by three lines in hyperopic eyes (to mean of $-0.04 \log$ MAR), ten lines in myopic eyes ( $-0.01 \log \mathrm{MAR})$, and one line in emmetropic eyes $(-0.07 \log$ MAR $)$ - again the smaller improvements were not unexpected in the emmetropic and hyperopic eyes. ${ }^{14}$ Although there were significant differences in UNVA and UDVA between each group preoperatively, no significant differences were observed 6 months after implantation; thus, the KAMRA inlay can be implanted after a LASIK procedure and the postoperative results appear similarly successful despite preoperative ametropia. ${ }^{14}$

Another case series by the same study group investigated the visual outcomes of the KAMRA inlay (model ACI7000PDT; again implanted in the nondominant eye) in 223 presbyopic patients who had previously undergone LASIK refractive surgery for emmetropia (mean spherical equivalent of $-0.18 \mathrm{D}$ in treated eye). After 6 months, the mean UNVA improved from Jaeger ( $\mathrm{J}) 8(\sim 0.50 \log \mathrm{MAR})$ to $\mathrm{J} 2(\sim 0.10$ $\log$ MAR) in the treated eye, but unfortunately binocular UNVA (BUNVA) was not reported. ${ }^{17}$ However, despite mean UDVA reducing slightly by one line from $-0.10 \log$ MAR to $0.00 \log$ MAR in the treated eye, the mean binocular UDVA (BUDVA) remained very good (-0.20 logMAR). ${ }^{17}$ Although $29 \%$ of patients had $>0.50 \mathrm{D}$ change, with a slight myopic shift compared to baseline, mean spherical equivalent refraction remained stable. ${ }^{17}$ Patient satisfaction of their visual status $(1=$ least, $7=$ most satisfied $)$ without reading glasses under bright light conditions improved significantly compared to baseline for all near (reading newspaper: $3.3 \pm 2.1$ to $5.0 \pm 1.4$; reading stock price on medicine bottle: $1.5 \pm 1.1$ to $4.1 \pm 1.8$ ) and intermediate tasks (reading the computer screen: $2.8 \pm 1.7$ to $5.6 \pm 1.2$ ) examined. ${ }^{17}$

Two-year follow-up of the efficacy of this inlay has also been investigated in 24 emmetropic presbyopes who underwent monocular implantation in the nondominant eye. In this study, the mean UNVA improved from $0.40 \operatorname{logMAR}$ to $0.10 \log$ MAR in the treated eye, with $83 \%$ achieving 0.10 $\log$ MAR or better. ${ }^{18}$ Mean unaided intermediate visual acuity (UIVA) improved from $0.20 \log$ MAR to $0.10 \log$ MAR, but UDVA decreased by one line compared to baseline in the treated eye ( $-0.10 \log$ MAR to $0.00 \log$ MAR). However, this is considered very good acuity and BUDVA remained stable $(-0.10 \log$ MAR over the 2 -year period $) .{ }^{18}$
Longer-term studies have also been reported, but mainly with the previous (original) version of the KAMRA implant (model ACI7000). This implant is slightly thicker $(10 \mu \mathrm{m})$ than the current design and has fewer porosity holes (1,600 holes $25 \mu \mathrm{m}$ in diameter). In a prospective cohort study, 32 naturally emmetropic patients who underwent implantation in the nondominant eye achieved mean UNVA of J2 ( 0.10 logMAR) after 2 years in the treated eye compared to J7/J8 ( 0.48/0.50 logMAR) preoperatively, with $96.9 \%$ of patients reading $\mathrm{J} 3(\sim 0.18 \log \mathrm{MAR})$ or better. ${ }^{19}$ Mean BUNVA also improved significantly from J6 $(\sim 0.40$ $\log$ MAR) preoperatively to J1 ( 0.00 $\log$ MAR). Mean UIVA improved from $0.30 \log$ MAR to $0.10 \log$ MAR in the treated eye and from $0.20 \log$ MAR to $0.00 \log$ MAR binocularly, with $71.9 \%$ of patients achieving $0.00 \log$ MAR or better. ${ }^{19}$ Although there was no significant difference between preoperative $(-0.10 \log \mathrm{MAR})$ and postoperative $(-0.10 \log$ MAR $)$ BUDVA, six patients experienced a reduction to $0.10 \log$ MAR and two patients to $0.20 \log$ MAR. ${ }^{19}$ However, mean UDVA in the treated eye remained $0.00 \log$ MAR over the 2 -year follow-up period. ${ }^{19}$ The same study group also reported at 3 years postoperatively on the same patient cohort. Mean UNVA was J1 ( $\sim 0.00 \log$ MAR), UIVA was $0.10 \log$ MAR, and UDVA was $0.00 \log$ MAR in the treated eye. ${ }^{20}$

Y1lmaz et al investigated the efficacy of the original inlay design up to 4 years postoperatively ( $n=22$ patients) in the natural and post-LASIK (to correct hyperopia) emmetropic presbyopes. ${ }^{21}$ Here, UNVA improved significantly from $\mathrm{J} 7(0.40 \log \mathrm{MAR})$ preoperatively to $\mathrm{J} 1(0.00 \log \mathrm{MAR})$ in the treated eye (mean improvement of $3.8 \pm 1.5$ lines; $96 \%$ reading J3 [ 0.18 logMAR] or better) at the last study visit. Compared to baseline, UDVA decreased, albeit statistically insignificantly, by one line ( $0.00 \log$ MAR to $0.10 \log$ MAR $)$ in the treated eye over the 4-year period. ${ }^{21}$ The longest follow-up with the KAMRA inlay (ACI7000) was recently reported by Dexl et al, where it was implanted in the nondominant eye of 32 natural emmetropic presbyopes. ${ }^{22}$ Mean UNVA improved significantly from J7/J8 ( 0.50 logMAR) preoperatively to $\mathrm{J} 1(\sim 0.00 \log \mathrm{MAR})$ at 1 year and remained stable over the next 3 years before tapering slightly to J3 $(\sim 0.18$ $\log$ MAR) after 5 years, with $74.2 \%$ of patients reading J3 $(\sim 0.18 \log \mathrm{MAR})$ or better in the treated eye. The BUNVA demonstrated the same pattern, but maintained consistently better acuity compared to monocular status, achieving a mean of J2 ( 0.10 logMAR) after 5 years, with $45.2 \%$ reading at $\mathrm{J} 1(\sim 0.00 \log \mathrm{MAR})$ or better. ${ }^{22}$ This pattern was also observed for UIVA in both monocular (0.20 logMAR; 
remaining similar to preoperative after 5 years) and binocular (0.10 logMAR after 5 years compared to $0.20 \log$ MAR preoperatively) states, with over 50\% reading $0.10 \log$ MAR or better. ${ }^{22}$ As observed in previous studies, mean UDVA decreased slightly from $-0.10 \log$ MAR preoperatively before tapering over the next 5 years to $0.10 \log$ MAR in the treated eye; however, mean BUDVA remained very good (-0.10 logMAR) with over 90\% achieving $0.00 \log$ MAR or better. ${ }^{22}$ In this study, acuity in the fellow, untreated eye was also measured preoperatively and at 5 years postoperatively. A similar decrease in UDVA was also observed. As a result, the authors attributed the loss of UDVA in both eyes to natural age-related hyperopic shift previously identified in the Beaver Dam and Liwan Eye Studies. ${ }^{23,24}$

In addition to measures of acuity, reading performance has also been assessed with the original KAMRA inlay. Dexl et al reported significant improvements in reading distance (reduced working distance), reading acuity at best working distance, and smallest print size in over a 2 -year period in 24 natural emmetropic presbyopes. ${ }^{25}$ However, although an increase in reading speed was also observed, this was not statistically significant. ${ }^{25}$

More recently, Tomita and Waring divided their patient cohort $(n=277)$ into three age groups $(40-49,50-59$, and 60-65 years) and performed simultaneous LASIK (to correct hyperopia) and KAMRA (ACI7000PDT) implantation to investigate the effect of age on safety and clinical outcomes over a 1-year period. The mean UNVA and UDVA were similar between groups, but the 60-65 years age group exhibited the largest gain in both outcomes at the final follow-up visit. ${ }^{26}$ Although this result was not unexpected, this group had lowest reduction in spectacle independence. The authors concluded that age should be taken into consideration during consultation in order to manage patient expectations postoperatively. ${ }^{26}$

\section{Safety and adverse events}

From the longer-term studies previously mentioned, it is apparent that UDVA in the treated eye and under binocular conditions becomes slightly compromised with the KAMRA inlay. However, in order to establish whether this is due to uncorrected residual ametropia or otherwise, measures of best corrected distance visual acuity (CDVA) have been evaluated. In the 3-year follow-up study by Seyeddain et al, although CDVA remained stable over time, $28.3 \%$ of patients lost one line and $3.1 \%$ lost two lines of acuity in the treated eye. Binocular CDVA was, however, stable and no patient lost a line of acuity during the follow-up period..$^{20}$ No inlays had to be explanted, but two had to be recentered after 6 months due to misalignment and no observable improvement in the near and intermediate acuity; once recentered, both patients subsequently achieved a significant improvement in these outcomes. ${ }^{20}$ One patient developed flap striae at 1 month and epithelial ingrowth at the flap interface, but were successfully resolved following surgical intervention. Of note, however, was the development of iron deposits in $56.2 \%$ of patients within a median interval of $18 \pm 9$ months after implantation. Although these deposits were not associated with visual or refractive outcomes, corneal topography revealed very small areas of flattening overlying the deposits. ${ }^{20}$ Corneal endothelial cell density decreased slightly $(5.73 \%)$ after 6 months, but further significant loss was not observed thereafter. ${ }^{20}$ The most common patient-reported symptoms at the final study visit (3 years) were night vision problems (40.6\% mild, 6.3\% moderate, and $15.6 \%$ severe cases) and halos $(34.4 \%, 25.0 \%$, and 3.1\%). Although dryness and glare were also reported, most cases were mild or moderate in nature. ${ }^{20}$

In the 4-year follow-up study, $27 \%$ of patients lost more than one line of CDVA, but mean CDVA did not change significantly from baseline (0.00 $\log \mathrm{MAR})$ to the final study visit $(0.00 \log$ MAR $)$ in the treated eye. ${ }^{21}$ Four patients had the inlay explanted: one at 6 weeks postimplantation due to the detection of a buttonhole flap, two at 3 months due to large refractive shifts $(-2.00 \mathrm{D}$ and $+3.00 \mathrm{D})$, and one at 17 months due to shallow implantation. All four of these patients were, however, successfully treated and no loss of monocular or binocular CDVA was observed. ${ }^{21}$ Complications reported included dry eye ( $\mathrm{n}=4$ treated eyes) and epithelial ingrowth $(n=5)$ related to LASIK, but it is not clear how the authors differentiated the cause between previous LASIK procedure and that of KAMRA implantation. In the 5-year follow-up study by Dexl et al, mean CDVA remained stable at $-0.10 \log$ MAR for the first 3 years before reducing to $0.00 \log$ MAR after 5 years in the treated eye, with $45.2 \%$ of patients losing one line and $22.6 \%$ losing two or more lines. ${ }^{22}$ A similar pattern emerged under binocular conditions, where mean CDVA reduced slightly from $-0.20 \log$ MAR preoperatively to $-0.10 \log$ MAR after 5 years, with $51.6 \%$ losing one line and $16.1 \%$ losing two or more lines. ${ }^{22}$ As observed in the study by Seyeddain et al (old inlay design ACI7000), iron deposits developed in 56.3\% of treated eyes at the 3-year follow-up and were associated with overlying corneal flattening; however, no further cases were observed for the remaining study period. ${ }^{20,22}$ The inlay was explanted from only one eye at the 36-month follow-up 
due to a hyperopic shift causing dissatisfaction with near and distance vision.

With the current inlay, Tomita et al reported no significant change in CDVA from baseline to 6 months postoperatively, although $14 \%$ of eyes lost one line of acuity in the treated eye. Despite this, all patients had monocular CDVA of 0.00 $\log$ MAR or better. ${ }^{17}$ Visual symptoms were also evaluated, albeit using a nonvalidated scale $(0=$ no symptoms, $7=$ very heavy symptoms); here, dryness, glare, halo, and night vision disturbances increased significantly, but were considered mild by the study authors. ${ }^{17}$ All patients were post-LASIK and therefore may be predisposed to such symptoms, which are typically associated with laser refractive procedures, but these symptoms should not be discounted, particularly when gaining consent for surgery. ${ }^{17,27}$ Similar results were also found by Seyeddain et al, where CDVA reduced by a mean of 2.5 letters from $-0.10 \log$ MAR to $0.00 \log$ MAR in the treated eye, with $16.7 \%$ of patients losing one line of acuity at the last follow-up (2 years); however, all of these patients achieved $0.00 \log$ MAR. ${ }^{18}$ No implants had to be recentered or explanted, and no ocular inflammation was observed during the study period. ${ }^{18}$ Adverse events included epithelial ingrowth at the pocket entrance at 1 month in one patient, epithelial iron deposits near the inlay margin at 18 months in one patient, while several others (number not reported) developed a thin hazy appearance at the outer and or inner rim of the inlay; however, they did not require treatment and were not associated with any visual or refractive outcomes. ${ }^{18}$ Further, endothelial cell count (ECC) and central corneal thickness (CCT) were not affected over the 2-year follow-up period. ${ }^{18}$ In another study of 24 emmetropic presbyopes who underwent monocular (nondominant) implantation, despite $16.7 \%$ losing one line and $4.1 \%$ losing two lines of CDVA in the treated eye at the final (2-year) follow-up, over $95 \%$ achieved CDVA of $0.00 \operatorname{logMAR}$ and binocular CDVA $(-0.10 \log$ MAR $)$ remained stable over the entire study period. ${ }^{28}$ No deposits were observed in or on the cornea, ECC and CCT were unaffected, and no patient required the inlay to be recentered or explanted. ${ }^{28}$ Only one patient experienced epithelial ingrowth at the pocket entrance after 1 month but remained stable and required no intervention. ${ }^{28}$

Confocal microscopy studies describing the corneal appearance with the implant in situ and postexplantation have also been performed. Abboud et al found that the implant changed the normal structure of the cornea (decreased keratocyte density in the anterior stroma, loss of subbasal nerve plexus), but this did not result in visual complications. ${ }^{29}$ However, keratocyte activation, also observed in typical laser refractive surgery procedures, was observed and this was significantly correlated with reduced UNVA, corrected near visual acuity, and CDVA. ${ }^{29}$ Thus, using lower laser energy, creating a thicker flap, and applying intensive steroid therapy postoperatively are suggested as key for good visual outcomes and healing response. ${ }^{29}$

\section{Discussion}

With both the original and current designs of the KAMRA implant, the clinical studies have clearly demonstrated significant improvements in both near and intermediate visual acuity with a minimal impact on distance vision following monocular implantation in the nondominant eye of presbyopes. Although longer-term studies for the current design are not yet reported, it is likely that the reduced incidence of loss of UDVA and CDVA is a result of improved surgical technique and implant design. ${ }^{27}$ The ACI7000 inlay was implanted under a corneal flap 170-180 $\mu \mathrm{m}$ deep using a microkeratome or femtosecond laser, ${ }^{20-22}$ whereas the thinner ACI7000PDT inlay is implanted at least $220 \mu \mathrm{m}$ deep in a pocket created with a femtosecond laser, thus the latter is less likely to affect corneal topography and subsequent visual acuity. ${ }^{18}$ Not only does the pocket technique allow for better centration, it also requires a smaller incision such that fewer corneal nerves are cut and therefore reduces the likelihood of postoperative dry eye typically associated with laser refractive procedures. ${ }^{13,30}$ Using femtosecond laser over mechanical microkeratome to create corneal flaps or pockets has been shown to provide lower incidence of postoperative dry eye, faster visual recovery, better UDVA, and more predictable incision depths. ${ }^{18,30,31}$ Although both inlay designs are microperforated to allow water and nutritional flow, ACI7000PDT is thinner $(6 \mu \mathrm{m}$ vs $10 \mu \mathrm{m})$ and has more holes $(8,400$ vs 1,600$)$, so is less likely to induce corneal thinning and epithelial decompensation. ${ }^{13,32}$ Indeed, only one in 20 patients developed epithelial iron deposits with the new design compared to over $56 \%$ with the ACI7000 inlay. ${ }^{18,20}$ However, in either case, these deposits neither interfered with vision nor were they associated with refractive outcomes. ${ }^{18}$

In cases where the inlays were explanted, all resolved without sequelae and without significant impact on distance vision; ${ }^{2}$ indeed, Alio et al reported that the KAMRA inlay is safe to remove and removal has minimal impact on corneal topography and aberrometry during and after recovery if explanted before 6 months. ${ }^{33}$ Thereafter, the changes in corneal topography may remain permanent. ${ }^{33}$ Despite decentration as little as $0.5 \mathrm{~mm}$ significantly affecting retinal image quality, recentration can be performed easily with subsequent 
improvements in near and distance acuity. ${ }^{14,20}$ Another major advantage of this surgery is that it is additive such that where the KAMRA inlays are removed, future options for presbyopia correction, including corneal approaches, are still available to the patient. ${ }^{32}$

Given that the inlay relies upon the pinhole effect to achieve improvement in near vision, the effect of pupil size on clinical outcome has been investigated, as pupil size is well known to influence optical image quality based on the amount of visual aberrations that pass in the eye after refractive surgery. ${ }^{34-36}$ An optical simulation of the KAMRA implant has shown that for combinations of pupil sizes and field angles, image brightness on the retina may be attenuated up to $60 \%$ due to its central position in front of the pupil and opaque nature, and it is predicted that this vignetting effect may lead to a clinically relevant reduction in contrast sensitivity. ${ }^{37}$ However, in a study of 584 actual KAMRA inlay treated eyes (584 patients), Tomita et al report that pupil size had no impact on visual acuity after implantation. There was no statistically significant difference between uncorrected and distance-corrected near visual acuity for both mesopic and photopic size groups. ${ }^{38}$ One study has reported a statistically significant reduction in contrast sensitivity in eyes with KAMRA inlays compared to preoperative measurements after 24 months in photopic and mesopic conditions, but these were found at higher spatial frequencies and the measures were within the range of the normal population. ${ }^{18}$ Another paper published by Vilupuru et al reported no loss of binocular contrast in either photopic or mesopic conditions for a series of 507 patients implanted monocularly with the KAMRA inlay. This same study also compared contrast sensitivity results for KAMRA inlay subjects to subjects treated with bilateral multifocal or accommodating intraocular lenses. Under all conditions, the KAMRA inlay patients demonstrated better contrast sensitivity than patients with the tested lenses. ${ }^{39}$

\section{Conclusion}

The KAMRA inlay is a safe and effective clinical procedure for the treatment of presbyopia, where significant improvement in near and intermediate visual acuity and function has been reported in several large and long-term follow-up studies. Although distance visual acuity has been compromised in some patients, the reductions were not clinically significant. Iron deposits within the corneal epithelium have been observed, but these are not considered to affect vision, and the incidence has reduced with improvements in surgical methods and the inlay design itself. Further studies with the latest KAMRA inlay are required to establish the longer-term safety and clinical stability of visual acuity.

\section{Disclosure}

The authors report no conflicts of interest in this work. The figures were supplied courtesy of AcuFocus Inc. (Irvine, CA, USA).

\section{References}

1. Pau H, Kranz J. The increasing sclerosis of the human lens with age and its relevance to accommodation and presbyopia. Graefes Arch Clin Exp Ophthalmol. 1991;229(3):294-296.

2. Glasser A, Campbell MC. Presbyopia and the optical changes in the human crystalline lens with age. Vision Res. 1998;38(2):209-229.

3. Heys KR, Truscott RJ. The stiffness of human cataract lenses is a function of both age and the type of cataract. Exp Eye Res. 2008;86(4): 701-703.

4. Strenk SA, Semmlow JL, Strenk LM, Munoz P, Gronlund-Jacob J, DeMarco JK. Age-related changes in human ciliary muscle and lens: a magnetic resonance imaging study. Invest Ophthalmol Vis Sci. 1999; 40(6):1162-1169.

5. Strenk SA, Strenk LM, Semmlow JL. High resolution MRI study of circumlental space in the aging eye. J Ref Surg. 2000;16(5):659-660.

6. Strenk SA, Strenk LM, Koretz JF. The mechanism of presbyopia. Prog Retin Eye Res. 2005;24(3):379-393.

7. Patel I, West SK. Presbyopia: prevalence, impact, and interventions. Community Eye Health. 2007;20(63):40-41.

8. Limnopoulou AN, Bouzoukis DI, Kymionis GD, et al. Visual outcomes and safety of a refractive corneal inlay for presbyopia using femtosecond laser. J Refract Surg. 2013;29(1):12-18.

9. Chayet A, Garza EB. Combined hydrogel inlay and laser in situ keratomileusis to compensate for presbyopia in hyperopic patients: one-year safety and efficacy. J Cataract Refract Surg. 2013;39(11): 1713-1721.

10. Garza EB, Gomez S, Chayet A, Dishler J. One-year safety and efficacy results of a hydrogel inlay to improve near vision in patients with emmetropic presbyopia. J Refract Surg. 2013;29(3):166-172.

11. Tabernero J, Schwarz C, Fernández EJ, Artal P. Binocular visual simulation of a corneal inlay to increase depth of focus. Invest Ophthalmol Vis Sci. 2011;52(8):5273-5277.

12. Lindstrom RL, MacRae SM, Pepose JS, Hoopes PC Sr. Corneal inlays for presbyopia correction. Curr Opin Ophthalmol. 2013;24(4): 281-287.

13. Dexl AK, Seyeddain O, Riha W, et al. One-year visual outcomes and patient satisfaction after surgical correction of presbyopia with an intracorneal inlay of a new design. J Cataract Refract Surg. 2012;38(2): 262-269.

14. Tomita M, Kanamori T, Waring GO, et al. Simultaneous corneal inlay implantation and laser in situ keratomileusis for presbyopia in patients with hyperopia, myopia, or emmetropia: six-month results. J Cataract Refract Surg. 2012;38(3):495-506.

15. Huseynova T, Kanamori T, Waring IV GO, Tomita M. Small-aperture corneal inlay in presbyopic patients with prior phakic intraocular lens implantation surgery: 3-month results. Clin Ophthalmol. 2013;7: 1683-1686.

16. Tabernero J, Artal P. Optical modelling of a corneal inlay in real eyes to increase depth of focus: optimum centration and residual defocus. J Cataract Refract Surg. 2012;38(2):270-277.

17. Tomita M, Kanamori T, Waring GO, Nakamura T, Yukawa S. Smallaperture corneal inlay implantation to treat presbyopia after laser in situ keratomileusis. J Cataract Refract Surg. 2013;39(6):898-905.

18. Seyeddain O, Bachernegg A, Riha W, et al. Femtosecond laser-assisted small-aperture corneal inlay implantation for corneal compensation of presbyopia: two-year follow-up. J Cataract Refract Surg. 2013;39(2): 234-241.

19. Seyeddain O, Riha W, Hohensinn M, Nix G, Dexl AK, Grabner G. Refractive surgical correction of presbyopia with the AcuFocus small aperture corneal inlay: two-year follow-up. J Refract Surg. 2010;26(10): $707-715$. 
20. Seyeddain O, Hohensinn M, Riha W, et al. Small-aperture corneal inlay for the correction of presbyopia: 3-year follow-up. J Cataract Refract Surg. 2012;38(1):35-45.

21. Y1lmaz ÖF, Alagöz N, Pekel G. Intracorneal inlay to correct presbyopia: long-term results. J Cataract Refract Surg. 2011;37(7):1275-1281.

22. Dexl AK, Jell G, Strohmaier C. Long-term outcomes after monocular corneal inlay implantation for the surgical compensation of presbyopia. $J$ Cataract Refract Surg. 2015;41(3):566-575.

23. Lee KE, Klein BE, Klein R, Wong TY. Changes in refraction over 10 years in an adult population: the Beaver Dam Eye study. Invest Ophthalmol Vis Sci. 2002;43(8):2566-2571.

24. He M, Huang W, Li Y, Zheng Y, Yin Q, Foster PJ. Refractive error and biometry in older Chinese adults: the Liwan eye study. Invest Ophthalmol Vis Sci. 2009;50(11):5130-5136.

25. Dexl AK, Seyeddain O, Riha W, Hohensinn M, Hitzl W, Grabner G Reading performance after implantation of a small-aperture corneal inlay for the surgical correction of presbyopia: two-year follow-up. J Cataract Refract Surg. 2011;37(3):525-531.

26. Tomita M, Waring GO. One-year results of simultaneous laser in situ keratomileusis and small-aperture corneal inlay implantation for hyperopic presbyopia: comparison by age. J Cataract Refract Surg. 2015;41(1): 152-161.

27. Melki SA, Azar DT. LASIK complications: etiology, management, and prevention. Surv Ophthalmol. 2011;46(2):95-116.

28. Dexl AK, Seyeddain O, Riha W. Reading performance and patient satisfaction after corneal inlay implantation for presbyopia correction: twoyear follow-up. J Cataract Refract Surg. 2012;38(10):1808-1816.

29. Abboud A, Javaloy J, Alió JL. Confocal microscopy evaluation of the corneal response following AcuFocus KAMRA inlay implantation. J Refract Surg. 2014;30(3):172-178.
30. Salomão MQ, Ambrósio R, Wilson SE. Dry eye associated with laser in situ keratomileusis: mechanical microkeratome versus femtosecond laser. J Cataract Refract Surg. 2009;35(10):1756-1760.

31. Kezirian GM, Stonecipher KG. Comparison of the IntraLase femtosecond laser and mechanical keratomes for laser in situ keratomileusis. J Cataract Refract Surg. 2004;30(4):804-811.

32. Arlt EM, Krall EM, Moussa S, Grabner G, Dexl AK. Implantable inlay devices for presbyopia: the evidence to date. Clin Ophthalmol. 2015;9:129-137.

33. Alió JL, Abbouda A, Huseynli S, Knorz MC, Durrie DS. Removability of a small aperture intracorneal inlay for presbyopia correction. J Refract Surg. 2013;29(8):550-556.

34. Martinez CE, Applegate RA, Klyce SD, McDonald MB, Medina JP, Howland HC. Effect of pupillary dilation on corneal optical aberrations after photorefractive keratectomy. Arch Ophthalmol. 1998;116(8): 1053-1062.

35. Miller JM, Anwaruddin R, Straub J, Schwiegerling J. Higher order aberrations in normal, dilated, intraocular lens, and laser in situ keratomileusis corneas. J Refract Surg. 2002;18(5):S579-S583.

36. Wang B, Ciuffreda KJ. Depth-of-focus of the human eye: theory and clinical implications. Surv Ophthalmol. 2006;51(1):75-85.

37. Langenbucher A, Goebels S, Szentmáry N, Seitz B, Eppig T. Vignetting and field of view with the KAMRA corneal inlay. Biomed Res Int. 2013;2013:154593.

38. Tomita M, Kanamori T, Waring GO, Huseynova T. Retrospective evaluation of the influence of pupil size on visual acuity after KAMRA inlay implantation. J Refract Surg. 2014;30(7):448-453.

39. Vilupuru S, Lin L, Pepose JS. Comparison of contrast sensitivity and through focus in small aperture inlay, accommodating intraocular lens, or multifocal intraocular lens subjects. Am J Ophthalmol. 2015;160(1):150-162.
Clinical Ophthalmology

\section{Publish your work in this journal}

Clinical Ophthalmology is an international, peer-reviewed journa covering all subspecialties within ophthalmology. Key topics include: Optometry; Visual science; Pharmacology and drug therapy in eye diseases; Basic Sciences; Primary and Secondary eye care; Patient Safety and Quality of Care Improvements. This journal is indexed on

Submit your manuscript here: http://www.dovepress.com/clinical-ophthalmology-journal

\section{Dovepress}

PubMed Central and CAS, and is the official journal of The Society of Clinical Ophthalmology (SCO). The manuscript management system is completely online and includes a very quick and fair peer-review system, which is all easy to use. Visit http://www.dovepress.com/ testimonials.php to read real quotes from published authors. 